\title{
Mecanismos de los efectos nocivos para la salud de la contaminación atmosférica proveniente de incendios forestales
}

\author{
BÁRBARA SANDOVAL D.*, TATIANA REYES R.** y MANUEL OYARZÚN G.**
}

Mechanisms of noxious effects of wildfire air pollution over human health

Wildfires represent a growing global public health issue, especially to the most vulnerable segment of the population (children, old people, pregnant women, patients with cardiovascular or respiratory diseases) exposed to smoke and other air borne contaminants generated from these events. In contrast to great cities' usual atmospheric pollution, that derives from forest fires differ in composition and its occurrence is sporadic and usually unpredictable. Exposure to atmospheric pollutants derived from forest fires has been associated to increased respiratory and cardiovascular morbidity, mediated by an inflammatory systemic response, oxidative stress and endothelial dysfunction. In people exposed to forest fire smoke an increased production of pro-inflammatory cytokines, endothelial activation and autonomic nervous system dysfunction has been observed, that leads to tissue injury, increased prothrombotic response, increased blood pressure and changes in heart rhythm. This review analyzes the mechanisms that have been involved in generating harmful health effects in humans exposed to inhaled particulate matter and gases steaming from wildfires.

Key words: Wildfires; particulate matter; cytokines; oxidative stress; lung injury; cardiovascular diseases.

\section{Resumen}

Los incendios forestales representan un problema creciente de la salud pública a nivel mundial, especialmente para la población más vulnerable (niños, ancianos, embarazadas y portadores de enfermedades cardiovasculares o respiratorias crónicas) expuesta al humo y a otros contaminantes aéreos. A diferencia de la contaminación atmosférica habitual de grandes urbes, aquella derivada de los incendios forestales tiene una composición diferente y su ocurrencia es esporádica y difícil de prever. La exposición a contaminantes atmosféricos derivados de incendios forestales se asocia a aumento de la morbilidad respiratoria y cardiovascular, mediada por una respuesta inflamatoria pulmonar y sistémica, estrés oxidativo y disfunción endotelial. En sujetos expuestos a humo de incendios forestales se ha observado un aumento en la producción de citoquinas pro-inflamatorias, activación endotelial y disfunción del sistema nervioso autónomo, que produce daño tisular, aumento de los mecanismos protrombóticos, aumento de la presión arterial y cambios en el ritmo cardiaco. Esta revisión analiza los mecanismos que han sido involucrados en generar efectos nocivos para la salud de seres humanos expuestos a material particulado y gases emanados de incendios forestales.

Palabras clave: Incendios forestales; material particulado; citoquinas; estrés oxidativo; daño pulmonar; enfermedades cardiovasculares.

\footnotetext{
* Ayudante-alumna, Escuela de Medicina, Facultad de Medicina, Universidad de Chile.

** Laboratorio de Investigaciones Respiratorias, Programa de Fisiopatología, Instituto de Ciencias Biomédicas, Facultad de Medicina, Universidad de Chile.
} 


\section{Introducción}

La contaminación atmosférica se define antropocéntricamente como la presencia en el aire atmosférico de compuestos químicos y/o biológicos que afectan la vida, la salud o el bienestar humano, que pueden provenir tanto de la actividad humana como de eventos naturales. Una de las fuentes de contaminación atmosférica, que se considera dentro de los desastres ambientales que pueden afectar al hombre, está constituida por la ocurrencia de incendios forestales. Los incendios forestales corresponden a una modalidad de exposición aguda a la contaminación atmosférica, cuya aparición y evolución es difícil de prever.

Según la Corporación Nacional Forestal (CONAF) en el período de un año (2018-2019) en Chile se han producido un total de 5.865 incendios forestales, afectando una superficie de 73.721 hectáreas. Estas cifras representan un aumento de 6 y $94 \%$ respecto al año anterior $(2016-2017)^{1}$. Las regiones con más superficie afectada en el último año (2018-19) han sido la Araucanía, Bío-Bío y Aysén. Sin embargo, son pocos los estudios que han analizado el efecto de estos incendios sobre la salud pública en nuestro país.

En otros lugares del mundo, se ha demostrado que este tipo de incendios aumenta la incidencia de enfermedades respiratorias y cardiovasculares en la población expuesta ${ }^{2}$. La variabilidad del efecto se ha relacionado a factores propios del incendio (extensión, duración, tipo de vegetación), atmosféricos (temperatura ambiental, velocidad y dirección del viento) y relacionados con la población. La magnitud de los efectos de esta exposición en la población depende de la presencia o no de comorbilidades. Además, muchas veces se suma la contaminación proveniente de incendios forestales a la contaminación atmosférica antropogénica de las grandes ciudades; por ello, no se ha logrado separar aún los efectos de la contaminación derivada de estos incendios de otras fuentes de contaminantes ${ }^{2}$.

Existen escasos estudios acerca de los mecanismos que intervienen en la patogenia de las enfermedades cuya frecuencia aumenta en la población expuesta a la contaminación aérea generada por los incendios forestales ${ }^{2}$. Se ha propuesto que estos mecanismos son provocados por el aumento de contaminantes atmosféricos tales como material particulado, monóxido de carbono, óxidos de nitrógeno y dióxido de azufre, a lo que se agrega el efecto del aumento de la temperatura ambiental. Creemos que es fundamental conocer los mecanismos por los cuales la contaminación atmosférica proveniente de incendios forestales produce alteraciones en la salud humana para estudiar su efecto en la población chilena.

Para esclarecer los mecanismos asociados a enfermedad en población expuesta a contaminación por incendios forestales se realizó una búsqueda bibliográfica de publicaciones (inglés/ español), entre 1996 y 2018, en revistas indexadas en PubMed, Google Scholar y SciELO, con los términos: 'contaminación atmosférica'; 'incendios forestales'; 'fisiopatología de incendios forestales'; 'efectos respiratorios'; 'efectos cardiovasculares'. Encontramos más de 100 publicaciones relacionadas, entre las cuales seleccionamos 44 publicaciones con evidencia de estudios "in vivo" e "in vitro" respecto a los mecanismos patogénicos de cada uno de estos contaminantes.

Además, analizaremos los mecanismos fisiopatológicos del daño producido por ozono, ya que este contaminante secundario que involucra en su generación, a múltiples contaminantes, se encuentra elevado en la tropósfera en el verano, época en que hay mayor frecuencia de incendios forestales en Chile.

\section{Absorción de partículas}

Cada día billones de partículas aéreas son inhaladas por las personas desde el ambiente; de estas, las menores a $10 \mu \mathrm{m}$ de diámetro aerodinámico tienen efectos biológicos en las personas expuestas $^{3}$.

Se han descrito diversos mecanismos por los cuales las partículas se depositan y penetran en el sistema respiratorio. Uno de ellos es la gravedad; es decir, aquellas características que dependen de la densidad de la partícula y la propia fuerza gravitacional. Otro mecanismo es el movimiento browniano, el cual depende del movimiento aleatorio de las partículas, aumentando el depósito de las partículas de menor tamaño. Un tercer mecanismo sería la sedimentación; en este mecanismo la gravedad y la resistencia del aire se anulan, y sólo influye en la absorción de las partículas la velocidad, diámetro y densidad de estas.

Dependiendo del diámetro aerodinámico de las partículas, estas pueden depositarse en distintas zonas del sistema respiratorio: Las partículas con diámetro mayor a $8 \mu \mathrm{m}$ impactan en el tracto respiratorio nasofaríngeo y los bronquiolos por inercia, las partículas menores a $3 \mu \mathrm{m}$ de diámetro aerodinámico se depositan en los alvéolos por mecanismos como movimiento browniano y sedimentación, y las partículas ultrafinas (diámetro aerodinámico $\leq 0,1 \mu \mathrm{m})$ pueden alcanzar 
fácilmente los alvéolos, para luego atravesar la barrera alvéolo-capilar y pasar a la circulación pulmonar y sistémica ${ }^{4,5}$.

\section{Contaminantes atmosféricos liberados en los incendios forestales}

Los contaminantes derivados de los incendios forestales provienen principalmente de la combustión de biomasa; por ello, sus principales componentes son el material particulado y gases derivados de la combustión como el dióxido y el monóxido de carbono (CO) y en menor proporción dióxidos de nitrógeno $\left(\mathrm{NO}_{2}\right)$ y de azufre $\left(\mathrm{SO}_{2}\right)$. En presencia de radiación ultravioleta, algunos de estos gases, como el $\mathrm{NO}_{2}$ y los compuestos orgánicos volátiles (COV), son generadores del ozono $\left(\mathrm{O}_{3}\right)$, por lo que se ha detectado aumento de $\mathrm{O}_{3}$ en incendios forestales estivales. Los análisis de la composición del material particulado $\leq 2,5 \mu \mathrm{m}\left(\mathrm{PM}_{2,5}\right)$ han detectado productos derivados de la combustión de la biomasa (levoglucano, oxalato, succinato, malonato, metoxifenoles, monosacáridos e hidrocarburos aromáticos policíclicos: HAPs), elementos derivados de la corteza terrestre (hierro, aluminio, calcio y sílice) y también aerosoles secundarios de nitratos, sulfatos y sales de amonio ${ }^{6}$.

La composición de los contaminantes liberados por los incendios forestales es menos compleja que la derivada de incendios de edificios, en que además de combustionar material sólido (biomasa), pueden combustionar líquidos inflamables, gas natural, butano y propano y estructuras metálicas y de material sintético. A modo de comparación en el incendio y destrucción de las torres gemelas de Nueva York, el material particulado estuvo compuesto por una compleja mezcla de restos de concreto, yeso, fibras de vidrio sintéticas, metales, especies iónicas y asbesto $^{7}$. Dado que el efecto de la contaminación atmosférica sobre los seres humanos depende de los elementos que la componen, y que estos componentes difieren entre incendios de edificios e incendios forestales, es comprensible que los efectos sobre la salud de la población expuesta también difieran entre ambas situaciones.

\section{Efectos del material particulado}

Los efectos inflamatorios observados tras la exposición a $\mathrm{PM}_{10}{ }^{8}$ también han sido atribuidos al $\mathrm{PM}_{2.5}{ }^{9}$, el cual al ser inhalado, puede depositarse directamente en los alvéolos ${ }^{5}$, en los cuales pro- voca una alteración de la fagocitosis alveolar por disminución de la viabilidad de los macrófagos alveolares, lo que explica la mayor susceptibilidad a infecciones que presentan los sujetos expuestos a $\mathrm{PM}_{2.5}{ }^{9}$.

Se ha demostrado que durante los incendios forestales aumenta la concentración de $\mathrm{PM}_{10}$, el cual se deposita directamente en el tejido pulmonar ${ }^{2}$; este contaminante provoca daño celular mediante la liberación de citoquinas, que aumentan significativamente su concentración, tanto local (en el tejido pulmonar) como sistémica. En estudios realizados en hombres sanos expuestos a altas concentraciones de material particulado in vivo, se ha detectado un aumento de la concentración de la proteína $\mathrm{C}$ reactiva en la sangre ${ }^{10}$. Más aún, voluntarios expuestos a altas concentraciones de material particulado, desarrollan una respuesta inflamatoria pulmonar con aumento de citoquinas como TNF- $\alpha$, IL-8, IL-1 $\beta$, aumento de neutrófilos y disminución de células alveolares viables en muestras de lavado broncoalveolar versus controles expuestos a una solución salina ${ }^{11}$. Otro estudio "in vitro" realizado en muestras de lavado broncoalveolar de ratas, expuestas a $\mathrm{PM}_{10}$ reveló que la exposición a altas concentraciones de $\mathrm{PM}_{10}$ se asocia con un alza de polimorfonucleares (PMN), aumento de la permeabilidad epitelial, de la actividad de la enzima lactato deshidrogenasa y de radicales libres, comprobando que la exposición a este contaminante induce a estrés oxidativo y reacción inflamatoria local ${ }^{12}$.

Se ha planteado que el aumento de la morbilidad cardiovascular en seres humanos previamente sanos o con patología cardiovascular crónica expuestos a contaminación atmosférica producida por incendios forestales, puede estar mediado por una respuesta inflamatoria sistémica inducida por las citoquinas liberadas por macrófagos alveolares activados. El aumento de la concentración del material particulado estimularía la producción de citoquinas en el pulmón que promueven una reacción inflamatoria sistémica, que involucra no sólo a células como leucocitos y plaquetas, sino también a agentes proinflamatorios, como GMCSF, IL-6, IL-1B, y sustancias protrombóticas ${ }^{8,13}$. También se ha descrito en episodios de contaminación por material particulado $\left(\mathrm{PM}_{10}\right), \mathrm{CO} \mathrm{y}$ $\mathrm{NO}_{2}$ aumento de proteínas pro-coagulantes en la respuesta inflamatoria de fase aguda del hígado como el fibrinógeno plasmático ${ }^{14}$.

En el desarrollo de la respuesta inflamatoria se produce una variación en las concentraciones de ciertas proteínas presentes en el plasma denominadas 'proteínas de fase aguda', entre las que se 
encuentran: Proteína $\mathrm{C}$ reactiva (PCR), amiloide A sérico, componentes del complemento y fibrinógeno que tienen un carácter adaptativo; también se generan las antiproteasas: $\alpha 1$-antitripsina y $\alpha 1$-antiquimotripsina y proteínas antioxidantes como Ceruloplasmina, Haptoglobina y Hemopexina. La medición de la concentración plasmática de estas proteínas puede ser útil en la monitorización del proceso ${ }^{15}$.

Lo anteriormente expuesto promueve la alteración sistémica del endotelio y específicamente, del endotelio pulmonar. Por otra parte, la exposición de seres humanos a elevadas concentraciones de material particulado estimula a la médula ósea a la liberación a la circulación de neutrófilos y monocitos, lo que se asocia con aumento de los niveles circulantes de IL-1 $\beta$ y de IL-6. Esta respuesta inflamatoria sistémica inducida por la inhalación de partículas provoca disfunción endotelial y una sobrerregulación de las moléculas de adhesión, de crucial importancia en el reclutamiento de leucocitos en las placas ateromatosas ${ }^{13}$ (Figura 1).

Un estudio de seguimiento electrocardiográfico a personas expuestas a altas concentraciones de material particulado detectó cambios en la frecuencia cardíaca que podrían explicarse por la disminución del tono vagal, tras la excitación de reflejos pulmonares del sistema nervioso autónomo, lo que podría ocasionar arritmias cardíacas y muerte ${ }^{16}$.

En conclusión, las alteraciones provocadas por el material particulado no solo producen un esta- do inflamatorio que afecta la función pulmonar, sino que también pueden determinar efectos en la función cardiovascular ${ }^{17}$, por daño directo al endotelio ${ }^{13}$. Existen estudios que asocian la exposición a material particulado y $\mathrm{NO}_{2}$ a los efectos cardiovasculares de un síndrome coronario agudo con elevación de ST en el ECG ${ }^{18}$.

Los efectos a largo plazo de la exposición, son aún desconocidos. Se puede especular que en el sistema cardiovascular podrían coadyuvar a los procesos de ateroesclerosis e hipertensión arterial sistémica ${ }^{13}$, en tanto que a nivel del sistema respiratorio a mediano plazo, podría ser la generación de hiperreactividad bronquial y tos crónica, y a más largo plazo podrían provocar reacciones fibróticas del intersticio pulmonar y potencialmente neoplasias pulmonares por sus eventuales componentes cancerígenos (HAPs).

\section{Mecanismos de alteraciones neurológicas y cerebrovasculares asociadas a la exposición de material particulado}

Diversas revisiones relacionan el aumento de la polución del aire con enfermedades cerebrovasculares. Existen estudios que relacionan el aumento del PM con los accidentes cerebro-vasculares $(\mathrm{ACV})^{19}$, planteándose tres posibles mecanismos por los cuales la exposición a material particulado genera los efectos ya mencionados.

Uno de estos mecanismos se basa en los efectos a nivel celular que produce el material

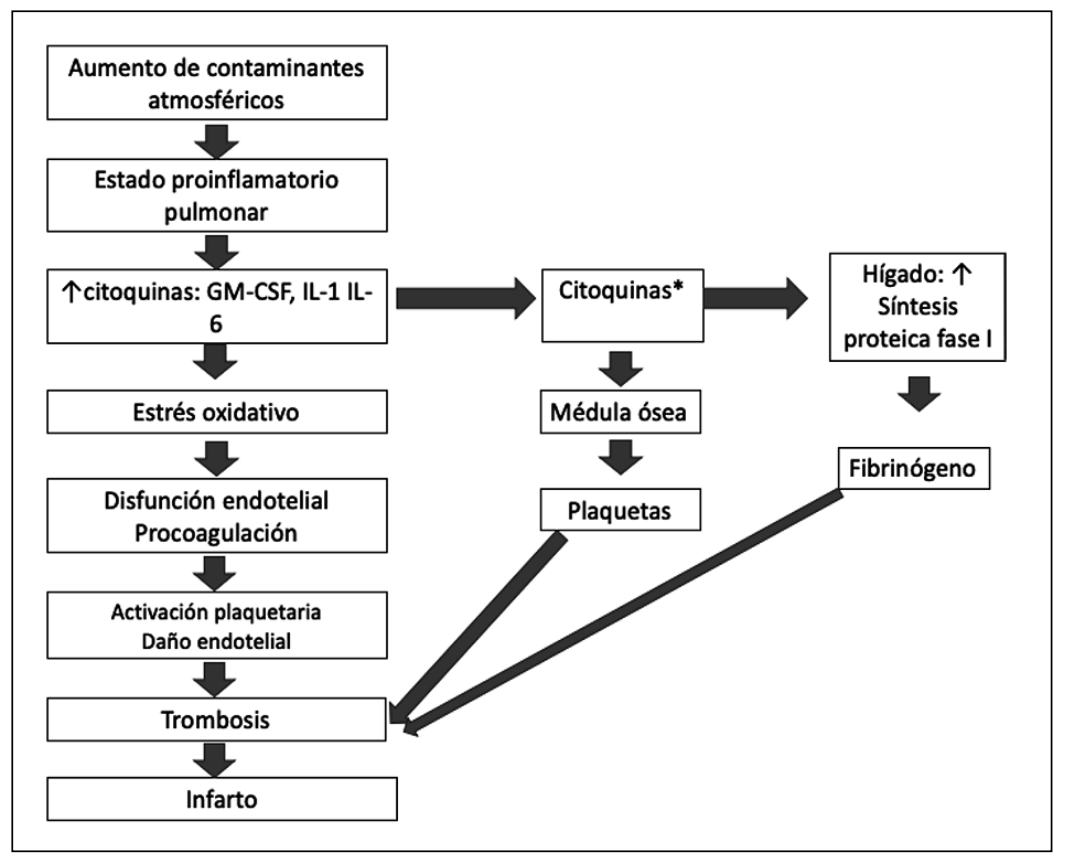

Figura 1. Mecanismos de daño inflamatorio del material particulado. GM-CSF: Factor estimulante de colonias de granulocitos y macrófagos, IL-1: Interleuquina 1, IL-6: Interleuquina 6. Síntesis Proteica fase 1 de la inflamación incluye diversas proteínas además del fibrinógeno y proteína $\mathrm{C}$ reactiva $(\mathrm{PCR})$. La señalización tanto a la médula ósea como al hígado, parte de la inflamación pulmonar, que determina el tipo y cantidad de citoquinas liberadas (Ver texto). 
particulado en el sistema respiratorio, lo cual determina efectos inflamatorios y protrombóticos, ya que aumenta la concentración de citoquinas como IL-1 e IL-6 y la estimulación de macrófagos y granulocitos ${ }^{20}$. En cuanto al aumento de elementos protrombóticos, Pekkanen y $\operatorname{cols}^{14}$ detectaron en la población londinense que el aumento de contaminación aérea por $\mathrm{NO}_{2}, \mathrm{CO}$ y $\mathrm{PM}_{10}$ se relaciona significativamente con el aumento de concentración de fibrinógeno plasmático, un reconocido factor de riesgo cardiovascu$\operatorname{lar}^{14}$. A su vez, en la población de Ausburgo, se ha detectado que la exposición a contaminación atmosférica por $\mathrm{SO}_{2}$ y material particulado se asocia significativamente con el aumento de la viscosidad sanguínea ${ }^{21}$; tanto el aumento de la viscosidad como del fibrinógeno podrían contribuir a aumentar el riesgo a presentar eventos cerebrovasculares en la población expuesta a contaminación atmosférica. Un ejemplo demostrativo de intoxicación por $\mathrm{CO}$ se ha comunicado, en relación con el gran incendio forestal ocurrido recientemente en Fuchal, Portugal ${ }^{22}$. Una cohorte de 37 casos de intoxicación aguda por $\mathrm{CO}$ derivado de incendios forestales fueron tratados por $72 \mathrm{~h}$ con oxigenación hiperbárica. El 78\% de estos intoxicados eran varones, 10 de ellos eran bomberos y 4 eran niños, además entre los intoxicados hubo dos mujeres embarazadas. Los síntomas más frecuentes fueron neurológicos (cefalea, mareos, náuseas, confusión y pérdida de conciencia). El nivel medio \pm rango intercurtílico de carboxihemoglobina fue de 3,7 $\pm 2,7 \%$ (valor normal: $1-2 \%$ en no fumadores). Al igual que en otros estudios la concentración de caboxihemoglobina no se correlacionó directamente con los síntomas neurológicos, probablemente porque estos también se deberían a que el $\mathrm{CO}$ inhibe la citocromo-oxidasa mitocondrial. Todos los pacientes recibieron oxigenoterapia desde el ingreso hasta el suministro de oxígeno hiperbárico. La persistencia de los síntomas fue la principal indicación de oxígeno hiperbárico. El tiempo medio para el oxígeno hiperbárico fue de $4,8 \mathrm{~h}$, a una presión de 2,5 atmósferas durante $90 \mathrm{~min}$, sin complicaciones mayores.

Un segundo mecanismo planteado es que el material particulado inhalado pasaría directamente a la circulación ${ }^{23}$, basándose en la capacidad de las nanopartículas de atravesar la barrera alvéolo-capilar, hecho que ha sido constatado en animales de experimentación ${ }^{23}$. Una vez que estas partículas llegan a estar en contacto con el endotelio pueden provocar efectos oxidativos e inflamatorios, como ocurre en el pulmón ${ }^{11}$. Los efectos inflamatorios de las nanopartículas se han demostrado también experimentalmente en animales ${ }^{24}$, en los cuales pueden provocar disfunción endotelial ${ }^{25}$ y aumentar la aterosclerosis ${ }^{24,26}$, lo cual podría explicar la asociación entre la exposición a altas concentraciones de material particulado y el aumento del espesor de las capas íntima y media en la carótida ${ }^{27}$. Además la exposición a material particulado se ha asociado a un aumento de la concentración de trombina en la circulación sanguínea ${ }^{28}$, y existen estudios que indican que pacientes diabéticos expuestos a material particulado presentan aumento de la actividad plaquetaria en estudios realizados en muestras sanguíneas ${ }^{29}$.

Un tercer mecanismo se basa en la capacidad de las partículas de reaccionar con los receptores neuronales del pulmón desencadenando un reflejo autonómico que provocaría aumento de la presión arterial y cambios en el ritmo cardíaco ${ }^{16,30}$.

El esquema presentado en la Figura 2 resume los tres mecanismos que explicarían la generación de daño cerebrovascular por exposición a material particulado. Varios de estos mecanismos participan en el daño al sistema cardiovascular explicado previamente.

\section{Efectos del ozono}

La liberación de contaminantes atmosféricos durante los incendios forestales altera la composición atmosférica y algunos de ellos como el $\mathrm{NO}_{2}$ y productos orgánicos volátiles pueden contribuir a la formación fotoquímica de ozono $\left(\mathrm{O}_{3}\right)^{30}$. Estudios internacionales han demostrado que durante los incendios forestales las concentraciones de $\mathrm{O}_{3}$ aumentan $^{31}$. En Chile se ha demostrado que durante los incendios forestales de Melipilla en el año 2014, los niveles de $\mathrm{O}_{3}$ aumentaron en Santiago, como también las concentraciones de $\mathrm{PM}_{10}$ y $\mathrm{CO}^{32}$.

En relación a los efectos que produce la exposición al ozono sobre el sistema respiratorio, los estudios realizados demuestran que la exposición aguda en voluntarios sanos se asocia a disminución de los valores espirométricos, síntomas de irritación traqueal, hiperreactividad bronquial e inflamación neutrofilica manifestada en el lavado broncoalveolar ${ }^{33}$. En exposiciones a largo plazo se ha encontrado daño de las células epiteliales, remodelación epitelial e intersticial y bronquiolización alveolar ${ }^{33}$.

En nuestro laboratorio hemos encontrado que la exposición aguda e intermitente en ratas a 0,5 ppm durante $4 \mathrm{~h}$ en dos días consecutivos produce inflamación neutrofílica de las vías aéreas, 


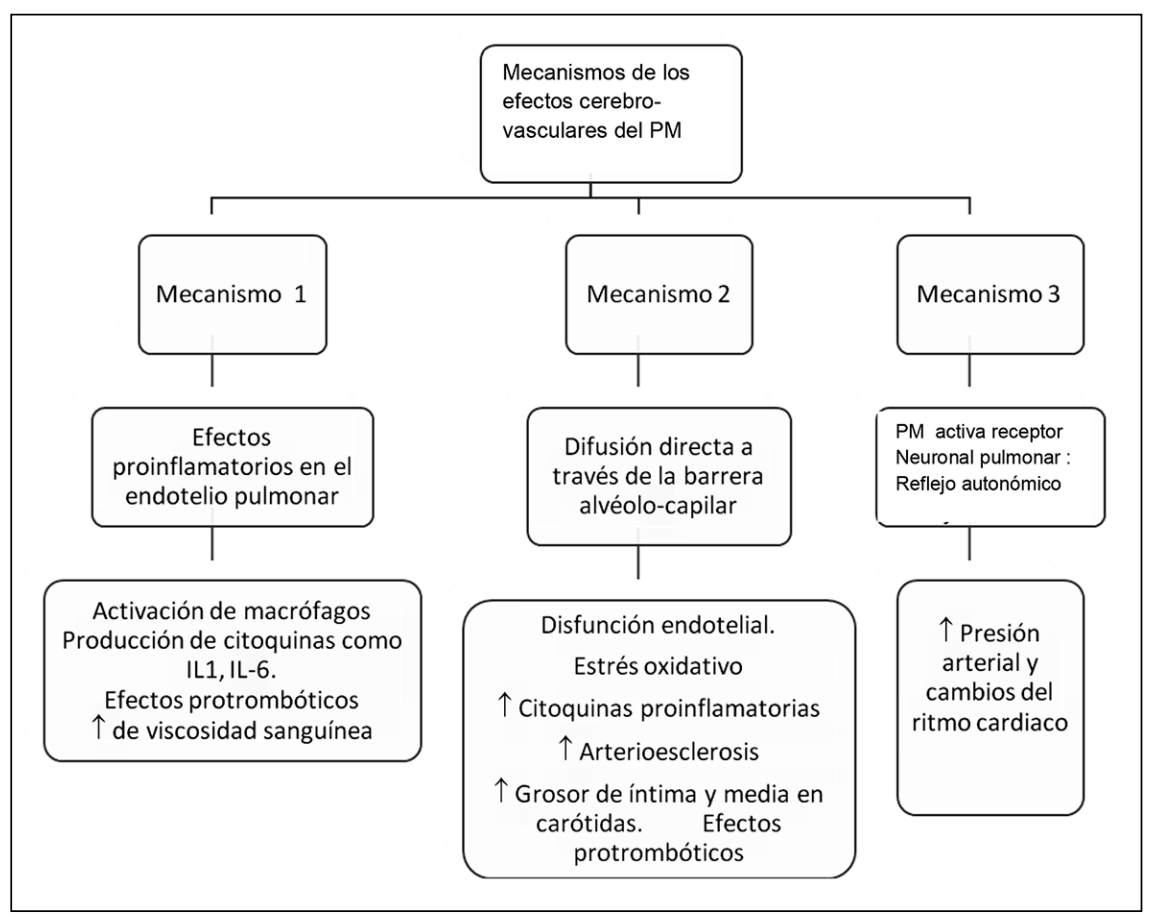

Figura 2. Mecanismos postulados para explicar los efectos cerebrovasculares del material particulado (PM). $\uparrow=$ aumento.

la que se exacerba con el ejercicio físico ${ }^{34}$. Estas alteraciones se explicarían por liberación de factores pro-inflamatorios como leucotrieno B4 y prostaglandina $\mathrm{F}_{2 \alpha}{ }^{34}$.

Las revisiones sobre los efectos cardiovasculares adversos relacionados con altos niveles de ozono ambiental son contradictorios, ya que hay estudios que evidencian que aumenta el riesgo cardiovascular, como también hay otros estudios que no muestran tal asociación ${ }^{35}$. Una de las hipótesis señala que este gas tiene un alto poder oxidativo determinando desequilibrio entre los agentes oxidantes y antioxidantes, generando un estado de estrés oxidativo que provocaría efectos adversos pulmonares y cardíacos ${ }^{36}$. Esta hipótesis concuerda con estudios in vivo en ratones expuestos a altas concentraciones de ozono, en los cuales se obtuvieron muestras de lavado broncoalveolar, que presentaban elevadas concentraciones de citoquinas inflamatorias como IL-6, IL-7 y TNF- $\alpha$, además de encontrar aumento del HIF-1 $\alpha$ (hipoxia-inducible factor 1 alpha $)^{37}$. En relación a un eventual efecto procoagulante del ozono, el estudio de Pekkanen y cols ${ }^{14}$ no logró demostrar asociación entre la concentración de ozono y el aumento de fibrinógeno en el plasma en la población londinense. Sin embargo, en ese mismo estudio ${ }^{14}$ si se demostró una asociación significativa entre el aumento de $\mathrm{NO}_{2}$ (contaminante precursor de $\mathrm{O}_{3}$ ) y el aumento de la concentración sanguínea de fibrinógeno ${ }^{14}$.

\section{Efectos del monóxido de carbono}

Los estudios existentes sobre los mecanismos de daño generados por la exposición al humo de los incendios forestales son escasos ${ }^{30}$. En ellos se ha demostrado que el aumento de contaminantes aéreos por combustión de biomasa, se asocia a aumento de leucocitos y polimorfonucleares (PMN) circulantes, y al aumento de citoquinas pro-inflamatorias (IL-6, IL-8) ${ }^{38}$. En estos estudios el CO se ha utilizado para monitorizar la magnitud de la intensidad de la exposición, siendo los efectos encontrados atribuidos principalmente al material particulado en el rango respirable $(\leq 10 \mu \mathrm{m})$.

La exposición a humo y $\mathrm{CO}$ en bomberos que combaten incendios forestales, se asoció a aumento de granulocitos en la expectoración y también en la circulación sistémica, indicando que hubo estimulación de la médula ósea; los autores atribuyeron estos hallazgos a la reacción inflamatoria ya descrita, y lo relacionaron con sintomatología de la vía respiratoria alta y baja en voluntarios $^{38}$. La estimulación de la médula ósea ha sido detectada en voluntarios adultos sanos expuestos al humo de incendios forestales en el Sureste asiático en 1997, en que se encontró una asociación significativa entre el aumento de $\mathrm{PM}_{10}$ y de polimorfonucleares sanguíneos, con un día de rezago ${ }^{39}$.

Como ya fue comentado el aumento de la 
concentración atmosférica de $\mathrm{CO}$ en ciudades se ha relacionado con el aumento de agentes protrombóticos como el fibrinógeno plasmático ${ }^{14} \mathrm{y}$ el aumento de viscosidad plasmática en la población expuesta $^{21}$. Por otra parte, la intoxicación aguda por CO derivada de incendios forestales produjo principalmente síntomas neurológicos ${ }^{22}$.

\section{Efectos del dióxido de azufre $\left(\mathrm{SO}_{2}\right)$}

La leucocitosis comunicada en sujetos durante incendios de biomasa no solo se ha atribuido a aumento de $\mathrm{PM}_{10}$, en concentraciones superiores a $125 \mu \mathrm{g} / \mathrm{m}^{3}$, sino también al aumento de $\mathrm{SO}_{2}$, siendo los polimorfonucleares (PMN) circulantes las células que muestran un mayor aumento en los períodos de incendios forestales ${ }^{39}$. Este mismo estudio señala que el aumento de producción de PMN por la médula ósea ante el aumento de $\mathrm{PM}_{10}$ es inmediato, en cambio al aumentar el $\mathrm{SO}_{2}$ estos efectos son más larvados ya que los polimorfonucleares aumentan con un rezago de 3 y 4 días respecto al aumento de $\mathrm{SO}_{2}$.

Investigaciones in vivo también han intentado relacionar la exposición a PM durante incendios forestales con estrés oxidativo, mediante la medición de 8-Oxo-2' deoxiguanosina (8-Oxo-dG) que es un nucleósido generado por la oxidación de $\mathrm{ADN}^{40}$. Esta molécula que se puede medir en la orina, se relaciona con diferentes procesos del estrés oxidativo y es considerada como un eventual biomarcador de riesgo de cáncer pulmonar. En estos estudios se ha encontrado que hay una relación entre la producción de 8-oxo-dG y la exposición a material particulado, según los años de ejercicio profesional de los bomberos ${ }^{41}$ (Figura 3).

En cuanto al $\mathrm{NO}_{2}, \mathrm{SO}_{2}$ y amonio las investigaciones han demostrado que la exposición a altas concentraciones de estos gases disminuye la viabilidad de las células pulmonares ${ }^{42}$.

\section{Especies reactivas del oxígeno como mediadores del daño inducido por incendios forestales}

Estudios "in vitro" sobre los efectos de la contaminación ambiental han demostrado que la exposición a material particulado estimula la producción de especies de oxígeno reactivas (ROS) no solo a nivel pulmonar, sino también a nivel cardíaco ${ }^{43}$, alterando su funcionamiento ${ }^{44}$.

Otras investigaciones han demostrado que la exposición a $\mathrm{PM}_{10}$ aumenta los niveles de IL-8 y de hemo-oxigenasa 1 (HMOX-1) $)^{45,46}$, siendo esto último un mecanismo de protección celular frente al estrés oxidativo en general, ya que como se ha mencionado la exposición a material particulado aumenta el estrés oxidativo ${ }^{36}$.

Estudios sobre exposición de partículas gruesas (diámetro aerodinámico entre 10 y $2,5 \mu \mathrm{m}$ ) en tejido pulmonar de ratas han demostrado aumento en la producción de TNF- $\alpha$, IL- 6 , neutrófilos y producción de especies reactivas del oxígeno (ROS). En cambio, la exposición a partículas ultrafinas no produjo efectos pulmonares lo cual podría estar relacionado con la diferente composición entre este tipo de partículas y/o con su diferente tiempo de permanencia en los espacios aéreos ${ }^{46,47}$.

\section{Conclusiones}

La alteración de los componentes atmosféricos producto de la ocurrencia de incendios forestales, genera repercusiones en la salud de las personas, provocando alteraciones respiratorias y cardiovasculares. Estos contaminantes constituidos fundamentalmente por partículas, ingresan por inhalación y el mecanismo por el cual se depositan en el organismo depende de su tamaño, destacando como mecanismos la misma gravedad, el movimiento browniano y la sedimentación. Los mecanismos de acción nociva de dichas partículas se pueden resumir en aumento de citoquinas pro-inflamatorias, lo que generará daño endotelial, como también la presencia de estrés oxidativo, siendo ambos mecanismos los responsables de las alteraciones de los sistemas respiratorio y cardiovascular.

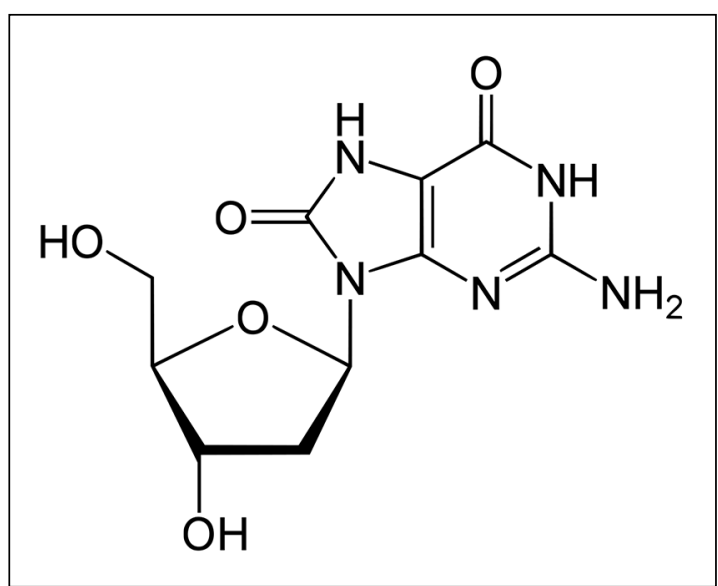

Figura 3. Estructura química de la 8-Oxo-2' deoxiguano$\operatorname{sina}(8-\mathrm{Oxo}-\mathrm{dG})^{30}$. 


\section{Bibliografía}

1.- CONAF. Número de incendios forestales y superficie afectada a la fecha. Disponible en: http:/www.conaf. $\mathrm{cl} /$ incendios-forestales/incendios-forestales-en-chile/ estadistica-de-ocurrencia-diaria/ (Fecha de la consulta: 20.03.2019).

2.- LIU JC, PEREIRA G, UHL SA, BRAVO MA, BELL ML. A systematic review of the physical health impact from non-occupational exposure to wildfire smoke. Environ Res 2015, 136: 120-32.

3.- HEYDER J. Deposition of Inhaled Particles in the Human Respiratory Tract and Consequences for Regional Targeting in Respiratory Drug Delivery. Proc Am Thorac Soc 2004, 1: 315-20.

4.- THOMAS, RJ. Particle size and pathogenicity in the respiratory tract. Virulence 2013; 4: 847-58.

5.- STUART BO. Deposition and Clearance of Inhaled Particles. Environ Health Perspect 1984, 55: 369-90.

6.- SILLANPÄÄ M, SAARIKOSKI S, HILLAMO R, PENNANEN A, MAKKONEN U, SPOLNIK Z, et al. Chemical composition, mass size distribution and source analysis of long-range transported wildfire smokes in Helsinki. Sci Total Environ. 2005; 350: 119-35.

7.- REIBMAN J, LEVY-CARRICK N, MILES T, FLYNN K, HUGHES C, CRANE M, et al. Destruction of the World Trade Center Towers. Lessons Learned from an Environmental Health Disaster. Ann Am Thorac Soc. 2016; 13: 577-83.

8.- VAN EEDEN SF, TAN WC, SUWA T, MUKAE H, TERASHIMA T, FUJI T, et al. Cytokines Involved in the Systemic Inflammatory Response Induced by Exposure to Particulate Matter Air Pollutants $\left(\mathrm{PM}_{10}\right)$. Am J Respir Crit Care Med 2001; 164: 826-30.

9.- XING Y-F, XU Y-H, SHI M-H, LIAN Y-X. The impact of PM2.5 on the human respiratory system. J Thorac Dis 2016; 8: E69-74.

10.- PETERS A, FRÖHLICH M, DÖRING A, IMMERVOLL T, WICHMANN HE, HUTCHINSON WL, et al. Particulate air pollution is associated with an acute phase response in men: Results from the MONICAAugsburg study. Eur Heart J 2001, 22: 1198-204.

11.- GHIO AJ, DEVLIN RB. Inflammatory lung injury after bronchial instillation of air pollution particles. Am J Respir Crit Care Med 2001; 164: 704-8.

12.- LI XY, GILMOUR PS, DONALDSON K, MACNEE W. Free radical activity and pro-inflammatory effects of particulate air pollution $\left(\mathrm{PM}_{10}\right)$ in vivo and in vitro, Thorax 1966; 51: 1216-22.

13.- VAN EEDEN SF, YEUNG A, QUINLAM K, HOGG JC. Systemic response to ambient particulate matter: relevance to chronic obstructive pulmonary disease. Proc Am Thorac Soc 2005; 2: 61-7.

14.- PEKKANEN J, BRUNNER EJ, ANDERSON HR, TIITTANEN P, ATKINSON RW. Daily concentrations of air pollution and plasma fibrinogen in London, Occup
Environ Med 2000; 57: 818-22.

15.- ECKERSALL PD. Acute phase proteins as markers of infection and inflammation: monitoring animal health, animal welfare and food safety. Irish Vet J 2000; 53: 307-11.

16.- POPE CA 3rd, VERRIER RL, LOVETT EG, LARSON AC, RAIZENNE ME, KANNER RE, et al. Heart rate variability associated with particulate air pollution. Am Heart J 1999, 138: 890-9.

17.- ROMÁN O, PRIETO MJ, MANCILLA P, ASTUDILLO P, DUSSAUBAT AM, MIGUEL C, et al. Daño cardiovascular por material particulado del aire. Puesta al día. Rev Med Chile 2009; 137: 1217-24.

18.- ARGACHA JF, COLLART P, WAUTERS A, KAYAERT P, LOCHY S, SCHOORS D, et al. Air pollution and ST-elevation myocardial infarction: A case-crossover study of the Belgian STEMI registry 2009-2013. Int J Cardiol 2016; 223: 300-5.

19.- FRANCHINI M, MANNUCCI PM. Thrombogenicity and cardiovascular effects of ambient air pollution. Blood 2011; 118: 2405-12.

20.- MILLS NL, DONALDSON K, HADOKE PW, BOON NA, MACNEE W, CASSEE FR, et al. Adverse cardiovascular effects of air pollution, Nat Clin Pract Cardiovasc Med 2009 6: 36-44.

21.- PETERS A, DÖRING A, WICHMANN H, KOENIG W. Increased plasma viscosity during an air pollution episode: a link to mortality? Lancet 1997; 349 (9065): 1582-87.

22.- SANTOS LR, ALVES-CORREIA M, CÂMARA M, LÉLIS M, CALDEIRA C, BRAZÃO ML, et al. Multiple Victims of Carbon Monoxide Poisoning in the Aftermath of a Wildfire: A Case Series. Acta Med Port 2018; 29: 31: 146-51.

23.- NEMMAR A, VANBILLOEN H, HOYLAERTS MF, HOET PHM, VERBRUGGEN A, NEMERY B. Brief Communication Passage of Intratracheally Instilled Ultrafine Particles from the Lung into the Systemic Circulation in Hamster, Am J Respir Crit Care 2001; 164: 1665-8.

24.- SUN Q, WANG A, JIN X, NATANZON A, DUQUAINE D, BROOK RD. Acceleration of Atherosclerosis and Vascular Inflammation in an Animal Model. JAMA 2015; 294: 3003-10.

25.- WOLD LE, YING Z, HUTCHINSON KR, VELTEN M, GORR MW., VELTEN C, et al. Cardiovascular Remodeling in Response to Long-Term Exposure to Fine Particulate Matter Air Pollution. Cir Heart Fail 2012; 5: 452-61.

26.- OHGAMI A, VINCENT R, VAN EEDEN SF. Particulate Air Pollution Induces Progression of Atherosclerosis on mortality statistics suggest that the effect of increasing. J Am Coll Cardiol 2002, 39: 935-42.

27.- ADAR SD, SHEPPARD L, VEDAL S, POLAK JF, SAMPSON PD, DIEZ ROUX AV, et al. Fine Particulate Air Pollution and the Progression of Carotid Intima- 
Medial Thickness: A Prospective Cohort Study from the Multi-Ethnic Study of Atherosclerosis and Air Pollution. PLoS Med 2013; 10 (4): e1001430.

28.- EMMERECHTS J, JACOBS L, KERCKHOVEN SVAN, LOYEN S. Air pollution-associated procoagulant changes: the role of circulating microvesicles, $\mathrm{J}$ Thromb Haemost 2012; 10: 96-106.

29.- JACOBS L, EMMERECHTS J, MATHIEU C, HOYLAERTS MF, FIERENS F, HOET PH. Air PollutionRelated Prothrombotic Changes in Persons with Diabetes, Environ Health Perspect 2010; 118: 191-6.

30.- CASCIO W. Wildland fire smoke and human health. Sci Total Environ 2018; 624: 586-95.

31.- BOSSIOLI E, TOMBROU M, KARALI A, DANDOU A, PARONIS D, SOFIEV M. Ozone production from the interaction of wildfire and biogenic emissions: A case study in Russia during spring 2006. Atmos Chem Phys 2012; 12: 7931-53.

32.- RUBIO MA, LISSI E, GRAMSCH E, GARREAUD RD. Effect of nearby forest fires on ground level ozone concentrations in Santiago, Chile. Atmosphere 2015; 6: 1926-38.

33.- OYARZÚN M. Efectos del ozono troposférico en el sistema respiratorio. En: Cabrera S, Lissi G y Honeymann J Eds. Radiación ultravioleta y salud. Editorial Universitaria. 2005; págs 285-95.

34.- OYARZÚN M, DUSSAUBAT N, MILLER ME, LÓPEZ M, MÉNDEZ G, MIRANDA J. El ejercicio físico aumenta el daño pulmonar inducido por la exposición aguda e intermitente a 0,5 ppm de ozono en ratas juveniles. Rev Chil Enferm Respir 2013; 29: 141-8.

35.- MAITRE A, BONNETERRE V, HUILLARD L, SABATIER P, DE GAUDEMARIS R. Impact of urban atmospheric pollution on coronary disease. Eur Heart $\mathrm{J}$ 2006; 27: 2275-84

36.- KELLY FJ. Oxidative stress: its role in air pollution and adverse health effects. Occup Environ Med 2003; 60: 612-6.

37.- WIEGMAN CH, LI F, CLARKE CJ, JAZRAWI E, KIRKHAM P, BARNES PJ, et al. A comprehensive analysis of oxidative stress in the ozone-induced lung inflammation mouse model. Clinical science 2014; 126 : 425-40.

38.- SWISTON JR, DAVIDSON W, ATTRIDGE S, LI GT, BRAUER M, VAN EEDEN SF. Wood smoke exposure induces a pulmonary and systemic inflammatory response in firefighters. Eur Respir J 2008; 32: 129-38.

39.- TAN WC, QIU D, LIAM BL, TZE PN, LEE SH, VAN EEDEN SF, HOGG JC. The Human Bone Marrow Response to Acute Air Pollution Caused by Forest Fires. Am J Respir Crit Care Med 2000; 161 (4): 1213-7.

40.- PATRA A, NAGY LD, ZHANG Q, SU Y, MÜLLER L, GUENGERICH FP, et al. Kinetics, structure, and mechanism of 8-oxo-7,8-dihydro-2'-deoxyguanosine bypass by human DNA polymerase. J Biol Chem 2014; 289: 16867-82.

41.- ADETONA O, ZHANG J, HALL DB, WANG JS, VENA JE, NAEHER LP. Occupational exposure to woodsmoke and oxidative stress in wildland firefighters. Sci Total Environ 2013; 449: 269-75.

42.- BAKAND S, WINDER C, HAYES A. Comparative in vitro cytotoxicity assessment of selected gaseous compounds in human alveolar epithelial cells, Toxicol In Vitro 2007; 21: 1341-7.

43.- GURGUEIRA SA, LAWRENCE J, COULL B, KRISHNA MURTHY GG, GONZÁLEZ-FLECHA B. Rapid increases in the steady-state concentration of reactive oxygen species in the lungs and heart after particulate air pollution inhalation. Environ Health Perspect 2002; 110: 749-55.

44.- RHODEN CR, WELLENIUS GA, GHELFI E, LAWRENCE J, GONZÁLEZ-FLECHA B. PM-induced cardiac oxidative stress and dysfunction are mediated by autonomic stimulation. Biochim Biophys Acta 2005; 1725: 305-13.

45.- OROZCO-IBARRA M, PEDRAZA-CHAVERRÍ J. Hemo oxigenasa: aspectos básicos y su importancia en el sistema nervioso central, Arch Neurocien (Mex) 2010; 15: 47-55.

46.- AKHTAR US, RASTOGI N, MCWHINNEY RD, URCH B, CHOW CW, EVANS GJ, et al. The combined effects of physicochemical properties of size-fractionated ambient particulate matter on in vitro toxicity in human A549 lung epithelial cells. Toxicol Rep 2014; 1: 145-56.

47.- KIM YH, TONG H, DANIELS M, BOYKIN E, KRANTZ QT, MCGEE J. Cardiopulmonary toxicity of peat wildfire particulate matter and the predictive utility of precision cut lung slices. Part Fibre Toxicol 2014; 11: 29.

Correspondencia a:

Dr. Manuel Oyarzún Gómez

Programa de Fisiopatología, Instituto de Ciencias

Biomédicas, Facultad de Medicina, Universidad de

Chile.

Avda. Salvador 486. Comuna de Providencia.

Santiago, Chile.

Email: moyarzun@med.uchile.cl 